\title{
On the minimal distance of a polynomial code
}

\author{
Péter Pál Pach $\|$ and Csaba Szabó" \\ Eötvös Loránd University, Department of Algebra and Number Theory, Budapest, Hungary \\ received $28^{\text {th }}$ April 2010, revised $16^{\text {th }}$ March 2011, accepted $17^{\text {th }}$ March 2011.
}

For a polynomial $f(x) \in \mathbb{Z}_{2}[x]$ it is natural to consider the near-ring code generated by the polynomials $f \circ x, f \circ$ $x^{2}, \ldots, f \circ x^{k}$ as a vectorspace. It is a 19 year old conjecture of Günter Pilz that for the polynomial $f(x)=$ $x^{n}+x^{n-1}+\cdots+x$ the minimal distance of this code is $n$.

The conjecture is equivalent to the following purely number theoretical problem. Let $\underline{m}=\{1,2, \ldots, m\}$ and $A \subset \mathbb{N}$ be an arbitrary finite subset of $\mathbb{N}$. Show that the number of products that occur odd many times in $\underline{n} \cdot A$ is at least $n$. Pilz also formulated the conjecture for the special case when $A=\underline{k}$. We show that for $A=\underline{k}$ the conjecture holds and that the minimal distance of the code is at least $n /(\log n)^{0.223}$.

While proving the case $A=\underline{k}$ we use different number theoretical methods depending on the size of $k$ (respect to $n$ ). Furthermore, we apply several estimates on the distribution of primes.

Keywords: near-ring code, minimal distance, prime

\section{Introduction}

For two finite subsets of the positive integers, $A$ and $B$ let $A * B=\{a b \mid a \in A, b \in B$ and $a b$ occurs odd many times in $A \cdot B\}$. In other words, if $A=\left\{a_{1}, \ldots, a_{k}\right\}$, then $A * B=a_{1} B \Delta \cdots \Delta a_{k} B$, where $\Delta$ denotes the symmetric difference. For a positive integer $m$ let $\underline{m}=\{1,2, \ldots, m\}$.

Conjecture 1 If $n, k$ are positive integers, then $|\underline{n} * \underline{k}| \geq n$.

For an arbitrary finite subset $A \subset \mathbb{N}$ it was proved that $|\underline{m} * A| \geq \pi(m)+1$, where $\pi(x)$ is the prime counting function, and the following conjecture was formulated (Pilz $(1992))$ :

Conjecture 2 Let $n$ be a positive integer and $K \subset \mathbb{N}$ be a finite set of integers. Then $|\underline{n} * K| \geq n$.

These purely number theoretical problems originate in the theory of near-ring codes. A near-ring can be described as a ring, where the addition is not necessarily commutative and only one of the distributive laws is required. A typical example is the near-ring of polynomials, where the addition is the usual polynomial addition, and multiplication is the composition of the polynomials. In this example the addition

\footnotetext{
†Email: ppp24ecs.elte.hu

‡Email: csaba@cs.elte.hu

1365-8050 @ 2011 Discrete Mathematics and Theoretical Computer Science (DMTCS), Nancy, France
} 
is commutative and only the right distributive law holds. Near-rings play an important role in combinatorics: They are used to construct block designs that give rise to efficient error correcting codes. For more information on these codes see Eggetsberger (2011), Pilz (1983) and Pilz (2011). A special and very interesting near-ring code is defined in the following way: Let $f \in \mathbb{Z}_{2}[x]$ be a polynomial and $C(f, k)$ the code generated (as a subspace) by the polynomials $f=f \circ x, f \circ x^{2}, \ldots, f \circ x^{k}$. For $f=x+x^{2}+\cdots+x^{n}$ a typical codeword is

$$
\sum_{i \in K} f \circ x^{i}=\sum_{j \in K * \underline{n}} x^{j}
$$

where $K$ is a finite subset of $\underline{k}$. As $C(f, k)$ is a linear code, its minimal distance is equal to the minimal weight of any nonzero codeword. Hence the minimum distance of $C(f, k)$ is the minimal value of $|\underline{n} * K|$ for some $K \subseteq \underline{k}$.

In this paper we settle Conjecture 1 , and prove that for arbitrary $n \in \mathbb{N}$ and finite set $K \subset \mathbb{N}$ we have $|\underline{n} * K| \geq c \cdot \frac{n}{\log ^{0.223} n}$ for some $c>0$. Note that the minimal distance in $C(f, k)$ depends heavily on $f$. If, for example, we start with $f(x)=x+x^{2}+x^{4}+\cdots+x^{2^{k}}$, then $f \circ x+f \circ x^{2}=x+x^{2^{k+1}}$, hence the minimal distance of the corresponding code is 2 .

The natural logarithm will be denoted by log through the whole paper.

\section{The general case}

Let us denote by $g(n)$ the minimal size of the set $\underline{n} * K$, where $K$ is a finite subset of the positive integers. In Pilz (1992) it is proved that $g(n) \geq \pi(n)+1$. In this section we improve this lower bound and prove that $g(n) \geq c \cdot \frac{n}{\log ^{0.223} n}$ for some $c>0$. The proof is based on the following lemma:

Proposition 1 For every positive integer $n$

$$
g(n) \geq \sum_{p \leq n} g\left(\left\lfloor n / p^{\alpha_{p}}\right\rfloor\right),
$$

where the sum goes over the primes less than $n$, and $\alpha_{p}$ is the largest integer such that $p^{\alpha_{p}} \leq n$.

Proof: Let $p \leq n$ be a prime and $K_{p} \subseteq K$ the subset of $K$ containing the elements that are divisible by the largest power of $p$ occuring as divisor of some element of $K$ (possibly $p^{0}=1$ ). Similarly, let $\underline{n}_{p} \subseteq \underline{n}$ be the set of elements of $\underline{n}$ that are divisible by $p^{\alpha_{p}}$. Note that $\underline{n}_{p}$ is never empty. By the maximality of the exponents of $p$ in $K_{p}$ and $\underline{n}_{p}$, for any $a \in \underline{n}_{p}, b \in K_{p}$ and $c \in \underline{n}, d \in K$ if $a b=c d$, then $c \in \underline{n}_{p}$ and $d \in K_{p}$ hold. We prove that for $p<q \leq n$ different primes $\underline{n}_{p} \cdot K_{p}$ and $\underline{n}_{q} \cdot K_{q}$ are disjoint. If for some $a \in \underline{n}$ and $b \in K$ we have $a b \in \underline{n}_{p} \cdot K_{p} \cap \underline{n}_{q} \cdot K_{q}$, then $a \in \underline{n}_{p} \cap \underline{n}_{q}$. Thus $a=p q d^{\prime}$, and $\bar{a}=p^{2} d^{\prime}<a$ is in $\underline{n}$. The exponent of $p$ in $\bar{a}$ is larger than the one in $a$, which is contradiction. Hence, $\underline{n} * K$ contains the disjoint union of the sets $\underline{n}_{p} \cdot K_{p}$ for $p \leq n$, so

$$
|\underline{n} * K| \geq \sum_{p \leq n}\left|\underline{n}_{p} * K_{p}\right|
$$


As $p^{\alpha_{p}} \leq n<p^{\alpha_{p}+1}$, clearly, $\underline{n}_{p}=\left\{p^{\alpha_{p}}, 2 p^{\alpha_{p}}, \ldots,\left\lfloor n / p^{\alpha_{p}}\right\rfloor p^{\alpha_{p}}\right\}$, where $\left\lfloor n / p^{\alpha_{p}}\right\rfloor<p$. Dividing by $p^{\alpha_{p}}$, we obtain that $\left|\underline{n}_{p} * K_{p}\right|=\left|\underline{\left\lfloor n / p^{\alpha_{p}}\right\rfloor} * K_{p}\right|$, thus by the definition of $g$ we get

$$
\left|\underline{n}_{p} * K_{p}\right|=\left|\underline{\left\lfloor n / p^{\alpha_{p}}\right\rfloor} * K_{p}\right| \geq g\left(\left\lfloor n / p^{\alpha_{p}}\right\rfloor\right) .
$$

By (1) we have

$$
g(n) \geq \sum_{p \leq n} g\left(\left\lfloor n / p^{\alpha_{p}}\right\rfloor\right)
$$

and this is what we wanted to prove.

Theorem 2 For every $\lambda>\lambda_{0}$ there exists a $c=c(\lambda)>0$ such that for every $n>1$

$$
g(n) \geq c \cdot \frac{n}{\log ^{\lambda} n},
$$

where $\lambda_{0}$ satisfies $\int_{0}^{1}\left(\frac{2}{y}\right)^{\lambda_{0}} \frac{1}{2-y} d y=1$. Note that $\lambda_{0} \sim 0.2223 \ldots$

Proof: Fix $1>\lambda>\lambda_{0}$. We claim that there exists some $c>0$ such that the inequality

$$
g(n) \geq c \cdot \frac{n}{\log ^{\lambda} n}
$$

holds for every $n>1$. The proof is by induction on $n$. First we discuss the induction step. Assume that (2) holds for $n<m$. Now, we show that it holds for $n=m$, as well. The value of $c$ will be chosen later. By Proposition 1 and the induction hypothesis:

$$
\begin{aligned}
& g(m) \geq \sum_{\sqrt{m}<p \leq m} g(\lfloor m / p\rfloor) \geq \sum_{\sqrt{m}<p<m / 2} c \cdot \frac{\lfloor m / p\rfloor}{\log ^{\lambda}(\lfloor m / p\rfloor)} \geq \\
& \geq \sum_{\sqrt{m}<p<m / 2} c \cdot \frac{\lfloor m / p\rfloor}{\log ^{\lambda}(\lfloor m / p\rfloor)} \geq \sum_{\sqrt{m}<p<m / 2} c \cdot \frac{m / p-1}{\log ^{\lambda}(\lfloor m / p\rfloor)}= \\
& \quad=\sum_{\sqrt{m}<p<m / 2} c \cdot \frac{m / p}{\log ^{\lambda}(\lfloor m / p\rfloor)}-\sum_{\sqrt{m}<p<m / 2} c \cdot \frac{1}{\log ^{\lambda}(\lfloor m / p\rfloor)} .
\end{aligned}
$$

In Rosser and Schoenfeld $(1962)$ it is proved that $\pi(m)<\frac{1.25506 m}{\log m}$ for every $m>1$, hence $\pi(m / 2)-$ $\pi(\sqrt{m}) \leq \pi(m)<1.5 \cdot \frac{m}{\log m}$. For the second term of the last line of 3 we obtain:

$$
\sum_{\sqrt{m}<p<m / 2} c \cdot \frac{1}{\log ^{\lambda}(\lfloor m / p\rfloor)} \leq \sum_{\sqrt{m}<p<m / 2} c \cdot \frac{1}{(\log 2)^{\lambda}} \leq 1.5 \cdot \frac{m}{\log m} \cdot \frac{c}{\log 2}=o\left(\frac{m}{\log ^{\lambda} m}\right),
$$

since $\lambda<1$. 
Now we estimate the main term. By Mertens' theorem, there exists a constant $M$ such that $\sum_{p \leq x} \frac{1}{p}=$ $\log \log x+M+o(1)$. Hence, for every $\varepsilon>0$ there exists $B=B(\varepsilon)$ such that for $B \leq a \leq b$

$$
\left|\sum_{a<p<b} \frac{1}{p}-\log \log b+\log \log a\right|<\varepsilon
$$

holds. For $m>2^{2 K}$ we have $m^{\frac{1}{2}+\frac{K-1}{2 K}}<m / 2$. Applying [5) to the interval $I_{h}=\left(m^{\frac{1}{2}+\frac{h-1}{2 K}}, m^{\frac{1}{2}+\frac{h}{2 K}}\right]$, where $h$ is an integer satisfying $1 \leq h \leq K-1$ we obtain that

$$
\sum_{p \in I_{h}} \frac{1}{p}>\log \frac{K+h}{K+h-1}-\varepsilon
$$

If $p \in I_{h}$, then $\log ^{\lambda}(m / p) \leq \log ^{\lambda}(m)\left(\frac{K-h+1}{2 K}\right)^{\lambda}$. Substituting into the main term of the last line of (3), omitting the integer parts and rearranging we get that

$$
\begin{aligned}
\sum_{\sqrt{m}<p<m / 2} c \cdot \frac{m / p}{\log ^{\lambda}(\lfloor m / p\rfloor)} & \geq c m \sum_{\sqrt{m}<p<m / 2} \frac{1 / p}{\log ^{\lambda}(m / p)} \geq \\
& \geq \frac{c m}{\log ^{\lambda} m} \sum_{h=1}^{K-1} \sum_{p \in I_{h}}\left(\frac{2 K}{K-h+1}\right)^{\lambda} \cdot \frac{1}{p} \geq \\
& \geq \frac{c m}{\log ^{\lambda} m}\left(\sum_{h=1}^{K-1}\left(\frac{2 K}{K-h+1}\right)^{\lambda} \log \frac{K+h}{K+h-1}-\varepsilon \sum_{h=1}^{K-1}\left(\frac{2 K}{K-h+1}\right)^{\lambda}\right) .
\end{aligned}
$$

Now we show that there exists some $K$ such that

$$
S_{K}=\sum_{h=1}^{K-1}\left(\frac{2 K}{K-h+1}\right)^{\lambda} \log \frac{K+h}{K+h-1}>1 .
$$

Let $f_{K}(y)=\left(\frac{2}{y}\right)^{\lambda} K \cdot \log \left(1+\frac{1}{K(2-y)}\right)$ and $f(y)=\left(\frac{2}{y}\right)^{\lambda} \cdot \frac{1}{2-y}$. The sequence of functions $f_{K}$ converges to $f$. Then

$$
S_{K}=\frac{f_{K}\left(\frac{1}{K}\right)+f_{K}\left(\frac{2}{K}\right)+\cdots+f_{K}\left(\frac{K}{K}\right)}{K}-\frac{f_{K}\left(\frac{1}{K}\right)}{K} .
$$

Let

$$
T_{K}=\frac{f\left(\frac{1}{K}\right)+f\left(\frac{2}{K}\right)+\cdots+f\left(\frac{K}{K}\right)}{K} .
$$

As $1>\lambda>\lambda_{0}$, the Riemann-sum $T_{k}$ converges to $\int_{0}^{1} f>1$. As $f_{K}\left(\frac{1}{K}\right) / K$ converges to 0 , it is easy to see that $S_{K}-T_{K}$ converges to 0 . Hence we can fix a $K$ such that $S_{K}>1$. Now, we can choose some 
$\varepsilon>0$ such that

$$
\eta=\sum_{h=1}^{K-1}\left(\frac{2 K}{K-h+1}\right)^{\lambda} \log \frac{K+h}{K+h-1}-1-\varepsilon \sum_{h=1}^{K-1}\left(\frac{2 K}{K-h+1}\right)^{\lambda}>0 .
$$

According to (4) there exists some $R$ such that if $R<m$, then

$$
\sum_{\sqrt{m}<p<m / 2} c \cdot \frac{1}{\log ^{\lambda}(\lfloor m / p\rfloor)} \leq \eta \cdot c \cdot \frac{m}{\log ^{\lambda} m} .
$$

By (3) and (7) we obtain that $g(m) \geq c \cdot \frac{m}{\log ^{\lambda} m}$ holds. If we choose $c>0$ such that 2 holds for $n \leq \max \left(2^{2 K}, B^{2}(\varepsilon), R\right)$, then 3 is gained.

\section{The case $K=\underline{k}$}

In this section we prove Conjecture 1 . We distinguish cases according to how large is $k$ according to $n$. The conjecture is true for $k \leq 8$. (Pilz (1992))

$$
\text { Case 1: } \quad 9 \leq k \leq 1.34 \cdot \log n
$$

We show that in this case the number of elements that occur exactly once in the product $\underline{n} \cdot \underline{k}$ is at least $n$. We shall need the following two observations.

Lemma 3 Let $n / 2<a \leq n$ and $b \in \underline{k}$ such that a is relatively prime to every number less than $k$. Then ab occurs once in $\underline{n} \cdot \underline{k}$.

Proof: Let us assume that $a_{1}, a_{2} \in \underline{n}$ and $b_{1}, b_{2} \in \underline{k}$ satisfy the conditions of the lemma, and $a_{1} b_{1}=a_{2} b_{2}$. Now, $a_{1} \mid a_{2} b_{2}$ and $a_{1}$ and $b_{2}$ are relatively prime, hence $a_{1} \mid a_{2}$. As $a_{1}>n / 2$ we have $2 a_{1}>n \geq a_{2}$, thus $a_{1}=a_{2}$, which implies $b_{1}=b_{2}$.

Lemma 4 If $k \geq 14$, then $\prod_{p \leq k}\left(1-\frac{1}{p}\right) \geq \frac{0.5}{\log k}$.

Proof: In Rosser and Schoenfeld (1962) it is shown that for $k>1$

$$
\frac{e^{-\gamma}}{\log k}\left(1-\frac{1}{\log ^{2} k}\right) \leq \prod_{p \leq k}\left(1-\frac{1}{p}\right)
$$

where $\gamma$ is the Euler constant. For $k>21$ by using the monotonicity of the logarithm function and $e^{-\gamma}>0.56$ we get that

$$
\frac{e^{-\gamma}}{\log k}\left(1-\frac{1}{\log ^{2} k}\right) \geq \frac{0.56}{\log k}\left(1-\frac{1}{\log ^{2} 22}\right)>\frac{0.5}{\log k} .
$$


For $14 \leq k \leq 21$ it is enough to check the statement when $k=14,17$ and 19 . For these numbers the values of $(\log k) \cdot \prod_{p \leq k}\left(1-\frac{1}{p}\right)$ are $0.506,0.511$ and 0.503 , respectively, hence the statement holds.

Proposition 5 Let $9 \leq k \leq 1.34 \cdot \log n$. Then $|\underline{n} * \underline{k}| \geq n$.

Proof: We show that there are at least $n$ products satisfying the conditions of Lemma 3 For this we need to estimate the number of integers between $n / 2$ and $n$ that are not divisible by a prime less than $k$. This number will be denoted by $D$. By the inclusion-exclusion principle

$$
D=n-\lfloor n / 2\rfloor+\sum_{h=1}^{r}(-1)^{h} \sum_{1 \leq i_{1}<\ldots<i_{h} \leq r}\left(\left\lfloor\frac{n}{p_{i_{1}} \ldots p_{i_{h}}}\right\rfloor-\left\lfloor\frac{n / 2}{p_{i_{1}} \ldots p_{i_{h}}}\right\rfloor\right),
$$

where $\pi(k)=r$ and $p_{1}, \ldots, p_{r}$ are the primes up to $k$. Applying $x-1<\lfloor x\rfloor \leq x$ to all $2^{r+1}$ terms of the right side we get that

$$
\begin{aligned}
D \geq n-n / 2+\sum_{h=1}^{r}(-1)^{h} \sum_{1 \leq i_{1}<\ldots<i_{h} \leq r}\left(\frac{n}{p_{i_{1}} \ldots p_{i_{h}}}-\frac{n / 2}{p_{i_{1}} \ldots p_{i_{h}}}\right) & -2^{r}= \\
& =\frac{n}{2} \prod_{p \leq k}\left(1-\frac{1}{p}\right)-2^{r} .
\end{aligned}
$$

If $k \geq 14$, Lemma 4 applies, and

$$
D \geq \frac{n}{2} \prod_{p \leq k}\left(1-\frac{1}{p}\right)-2^{r} \geq \frac{0.25 n}{\log k}-2^{r}
$$

As $k \leq 1.34 \log n$, for $k \geq 14$ we have the estimation

$$
2^{r}=2^{\pi(k)} \leq 2^{k / 2} \leq \frac{1}{100 \log k} \cdot e^{\frac{k}{1.34}} \leq \frac{n}{100 \log k}
$$

Hence, $D \geq \frac{0.24 n}{\log k}$. Using Lemma 3 we obtain $|\underline{n} * \underline{k}| \geq D k$. The function $x / \log x$ is monotone increasing on $[1, \infty)$, thus

$$
|\underline{n} * \underline{k}| \geq D k \geq \frac{0.24 k}{\log k} n \geq \frac{0.24 \cdot 14}{\log 14} n>n .
$$

For $9 \leq k \leq 13$ we have

$$
|\underline{n} * \underline{k}| \geq D k \geq\left(\frac{n}{2} \prod_{p \leq k}\left(1-\frac{1}{p}\right)-2^{\pi(k)}\right) k .
$$

For $10 \leq k \leq 13$ it is obtained by calculation that the right hand side is greater than $n$ if $n \geq e^{k / 1.34}$. For $k=9$ the inequality holds if $n>5040$. By brute force the statement can be checked for $k=9$ and $n \leq 5040$. Thus we obtained $|\underline{n} * \underline{k}|>n$. 
Case 2: $\quad 1.34 \cdot \log n \leq k \leq n-\frac{0.22 \cdot n}{\log n}$ and $n \geq 1410$.

Let $k_{1}=\max (k, n / 7)$ and $k_{1}<p \leq n$ a prime. As $k<p$, the set of elements of $\underline{n} * \underline{k}$, which are divisible by $p$ is $\{p, 2 p, \ldots,\lfloor n / p\rfloor p\} * \underline{k}$. This set has the same cardinality as the set $\lfloor n / p\rfloor * \underline{k}$. Now, $\lfloor n / p\rfloor \leq 6$, hence $|\lfloor n / p\rfloor * \underline{k}| \geq k$. It is easy to see that for $p>q>n / 7$ an element of $\underline{n} * \underline{k}$ cannot be divisible by both $p$ and $q$. Hence, $|\underline{n} * \underline{k}| \geq\left(\pi(n)-\pi\left(k_{1}\right)\right) k$.

At first, suppose that $k \leq n / 7$. By a theorem of Dusart Dusart(1999) for $x \geq 17$

$$
\frac{x}{\log x} \leq \pi(x) \leq \frac{x}{\log x}\left(1+\frac{1.2762}{\log x}\right)
$$

holds. Hence, $\pi(n)-\pi(n / 7) \geq 0.749 \cdot \frac{n}{\log n}$ for $n \geq 1410$. As $1.34 \cdot \log n \leq k$, we have

$$
|\underline{n} * \underline{k}| \geq 1.34 \cdot 0.749 \cdot n>n \text {. }
$$

Secondly, let us consider the case when $n / 7<k \leq n / 2$. As $\pi(n)-\pi(n / 2) \geq 7$,

$$
|\underline{n} * \underline{k}| \geq\left(\pi(n)-\pi\left(k_{1}\right)\right) k>7 \cdot n / 7=n .
$$

Finally, let $n / 2<k<n-\frac{0.22 \cdot n}{\log n}$. Then by the estimates in Dusart (1999) and Robin (1983) there are at least two primes between $k$ and $n$ if $n>90000$. It can be checked that this also holds for $n>1410$. Thus

$$
|\underline{n} * \underline{k}| \geq(\pi(n)-\pi(k)) k \geq 2(n / 2)=n .
$$

We continue with the case when $k$ is "large", that is, $n-\frac{0.4 \cdot n}{\log n+1.02} \leq k$. By calculation we have $n-\frac{0.4 \cdot n}{\log n+1.02} \leq n-\frac{0.22 \cdot n}{\log n}$ for $n \geq 4$.

Case 3:

$$
n-\frac{0.4 \cdot n}{\log n+1,02} \leq k \leq n \text { and } n>5000
$$

If $k=n$, then $\underline{k} \cdot \underline{n}=\{1, \ldots, n\} \cdot\{1, \ldots, n\}$. If $a \neq b$, then pairing $a b$ with $b a$ only the products of the form $a \cdot a$ are left, hence $\underline{n} * \underline{k}=\left\{1^{2}, 2^{2}, \ldots, n^{2}\right\}$. Thus

$$
|\underline{n} * \underline{k}|=n \text {. }
$$

Assume now that $k<n$. Then

$$
|\underline{n} * \underline{k}|=|(\underline{k} * \underline{k}) \Delta((\underline{n} \backslash \underline{k}) * \underline{k})|=|\underline{k} * \underline{k}|+|(\underline{n} \backslash \underline{k}) * \underline{k}|-2|(\underline{k} * \underline{k}) \cap((\underline{n} \backslash \underline{k}) * \underline{k})| .
$$

For the first term on the right side of (11) we have

$$
|\underline{k} * \underline{k}|=\left|\left\{1^{2}, 2^{2}, \ldots, k^{2}\right\}\right|=k .
$$


Lemma 6 For the second term of 11 we have

$$
|(\underline{n} \backslash \underline{k}) * \underline{k}| \geq 2 k-n .
$$

Proof: We use the following observation: If

$$
i \leq \frac{k}{n-k} \quad \text { and } \quad k+1 \leq j \leq n,
$$

then $i j$ appears exactly once in $(\underline{n} \backslash \underline{k}) \cdot \underline{k}$, so $i j \in(\underline{n} \backslash \underline{k}) * \underline{k}$. Let us assume that $i j=i^{\prime} j^{\prime}$ such that $1 \leq i^{\prime} \leq k$ and $k+1 \leq j^{\prime} \leq n$. If $i=i^{\prime}$, then $j=j^{\prime}$. If $i^{\prime}<i$, then $1 \leq i^{\prime} \leq \frac{k}{n-k}$ and $k+1 \leq j^{\prime} \leq n$. Now, changing the roles of $(i, j)$ and $\left(i^{\prime}, j^{\prime}\right)$ we may assume that $i<i^{\prime}$. As $i j=i^{\prime} j^{\prime}$, we have $\frac{i}{i^{\prime}}=\frac{j^{\prime}}{j}$ and

$$
\frac{i}{i^{\prime}} \leq \frac{i}{i+1} \leq \frac{\frac{k}{n-k}}{\frac{k}{n-k}+1}=\frac{k}{n}<\frac{k+1}{n} \leq \frac{j^{\prime}}{j}
$$

which is a contradiction. For $(\underline{n} \backslash \underline{k}) * \underline{k}$ we obtain that

$$
|(\underline{n} \backslash \underline{k}) * \underline{k}| \geq\left\lfloor\frac{k}{n-k}\right\rfloor(n-k) \geq\left(\frac{k}{n-k}-1\right)(n-k)=k-(n-k)=2 k-n .
$$

Now, we focus on the third term of (11).

Lemma 7 For the third second term of 111

$$
|(\underline{k} * \underline{k}) \cap((\underline{n} \backslash \underline{k}) * \underline{k})| \leq 0.431 \cdot k .
$$

holds.

Proof: It is enough to show that among the numbers $1^{2}, 2^{2}, \ldots, k^{2}$ at most $0.431 k$ many has a divisor in the interval $[k+1, n]$. Let $k+1 \leq m \leq n$ and $m=a_{m} b_{m}^{2}$, where $b_{m}^{2}$ is the largest square divisor of $m$. Since $a_{m}$ is squarefree, $m \mid i^{2}$ if and only if $a_{m} b_{m} \mid i$. Let $S$ denote the following upper bound of the number of elements of the set $\left\{1^{2}, 2^{2}, \ldots, k^{2}\right\}$ which have a divisor in $[k+1, n]$ :

$$
S=\sum_{m=k+1}^{n}\left\lfloor\frac{k}{a_{m} b_{m}}\right\rfloor \leq \sum_{m=k+1}^{n} \frac{k}{a_{m} b_{m}}=k \sum_{m=k+1}^{n} \frac{b_{m}}{m}
$$

Recall that $m=a_{m} b_{m}^{2}$, where $a_{m}$ is squarefree. Now, summing by $j=b_{m} \leq \sqrt{m}$ :

$$
S=k \sum_{j=1}^{\lfloor\sqrt{n}\rfloor} \sum_{\substack{j^{2}|m, k+1 \leq m \leq n,\\| \mu\left(m / j^{2}\right) \mid=1}} \frac{j}{m} \leq k \sum_{j=1}^{\lfloor\sqrt{n}\rfloor} j \sum_{\substack{j^{2} \mid m, k+1 \leq m \leq n}} \frac{1}{m}
$$


Rewrite $S=k\left(S_{1}+S_{2}\right)$, where

$$
S_{1}:=\sum_{j=1}^{\lfloor\sqrt{n} / 2\rfloor} j \sum_{\substack{j^{2} \mid m, k+1 \leq m \leq n}} \frac{1}{m} \quad \text { and } \quad S_{2}:=\sum_{j=\lfloor\sqrt{n} / 2\rfloor+1}^{\lfloor\sqrt{n}\rfloor} j \sum_{\substack{j^{2} \mid m, k+1 \leq m \leq n}} \frac{1}{m} .
$$

First, we give an upper bound for $S_{1}$.

\section{Lemma 8}

$$
S_{1} \leq\left(\frac{\log n}{2}+0.31\right)(\log n-\log k)+\frac{n+2 \sqrt{n}}{8 k}
$$

Proof: Let $r_{j}=\left\lceil\frac{k+1}{j^{2}}\right\rceil$ and $s_{j}=\left[\frac{n}{j^{2}}\right]$. Then

$$
S_{1}=\sum_{j=1}^{\lfloor\sqrt{n} / 2\rfloor} j \sum_{l=r_{j}}^{s_{j}} \frac{1}{l j^{2}}=\sum_{j=1}^{\lfloor\sqrt{n} / 2\rfloor} \frac{1}{j} \sum_{l=r_{j}}^{s_{j}} \frac{1}{l} .
$$

The function $\frac{1}{x}$ is a nonnegative decreasing function on $(0, \infty)$, hence we can estimate the inside sum by

$$
\sum_{l=r_{j}}^{s_{j}} \frac{1}{l} \leq \int_{r_{j}}^{s_{j}} 1 / x+\frac{1}{r_{j}}=\log s_{j}-\log r_{j}+\frac{1}{r_{j}}
$$

As $\frac{k}{j^{2}} \leq r_{j}$ and $s_{j} \leq \frac{n}{j^{2}}$ we have

$$
\log s_{j}-\log r_{j}=\log \frac{s_{j}}{r_{j}} \leq \log \frac{n / j^{2}}{k / j^{2}}=\log n-\log k .
$$

Substituting into 17] we obtain

$$
S_{1} \leq \sum_{j=1}^{\lfloor\sqrt{n} / 2\rfloor} \frac{1}{j}\left(\log s_{j}-\log r_{j}+\frac{1}{r_{j}}\right) \leq \sum_{j=1}^{\lfloor\sqrt{n} / 2\rfloor} \frac{1}{j}\left(\log n-\log k+\frac{j^{2}}{k}\right) .
$$

Since

$$
\sum_{j=1}^{\lfloor\sqrt{n} / 2\rfloor} \frac{1}{j} \leq \log \lfloor\sqrt{n} / 2\rfloor+1 \leq \frac{\log n}{2}-\log 2+1 \leq \frac{\log n}{2}+0.31 .
$$

and

$$
\sum_{j=1}^{\lfloor\sqrt{n} / 2\rfloor} j=\frac{\lfloor\sqrt{n} / 2\rfloor \cdot(\lfloor\sqrt{n} / 2\rfloor+1)}{2} \leq \frac{n+2 \sqrt{n}}{8},
$$

from the inequalities (18, 19 , 20) we get 16 . 
Now we give an upper bound for $S_{2}$.

\section{Lemma 9}

$$
S_{2} \leq\left(1+\frac{1}{\sqrt{2}}+\frac{1}{\sqrt{3}}\right) \cdot \frac{n-k}{2 \sqrt{k}} \cdot \frac{\sqrt{n}}{k}+\frac{3 \sqrt{n}}{k}<1.15 \cdot \frac{(n-k) \sqrt{n}}{k^{3 / 2}}+\frac{3 \sqrt{n}}{k} .
$$

Proof:

$$
S_{2}=\sum_{j=\lfloor\sqrt{n} / 2\rfloor+1}^{\lfloor\sqrt{n}\rfloor} \sum_{\substack{j^{2} \mid m, k+1 \leq m \leq n}} \frac{j}{m}
$$

In (22) for every $j$ we have

$$
n \geq j^{2} \geq(\lfloor\sqrt{n} / 2\rfloor+1)^{2}>\frac{n}{4} .
$$

Hence $m=j^{2}$ or $2 j^{2}$ or $3 j^{2}$. As $k<m \leq n$, for $m=i j^{2}(i=1,2,3)$ we get

$$
\sqrt{\frac{k}{i}}<j \leq \sqrt{\frac{n}{i}} \text { and } \frac{j}{m} \leq \frac{\sqrt{n}}{k} .
$$

For fixed $i$, the number of $j$ such that $m=i j^{2}$ is at most:

$$
\left\lceil\frac{\sqrt{n}-\sqrt{k}}{\sqrt{i}}\right\rceil=\left\lceil\frac{1}{\sqrt{i}} \cdot \frac{n-k}{\sqrt{n}+\sqrt{k}}\right\rceil \leq \frac{1}{\sqrt{i}} \cdot \frac{n-k}{2 \sqrt{k}}+1,
$$

thus

$$
S_{2} \leq\left(1+\frac{1}{\sqrt{2}}+\frac{1}{\sqrt{3}}\right) \cdot \frac{n-k}{2 \sqrt{k}} \cdot \frac{\sqrt{n}}{k}+\frac{3 \sqrt{n}}{k}<1.15 \cdot \frac{(n-k) \sqrt{n}}{k^{3 / 2}}+\frac{3 \sqrt{n}}{k},
$$

and this is what we wanted to show.

Summarizing the results, from (16) and 21) we obtain:

$$
\begin{aligned}
S=k\left(S_{1}\right. & \left.+S_{2}\right) \leq \\
& \leq k\left\{\left(\frac{\log n}{2}+0.31\right)(\log n-\log k)+\frac{n+2 \sqrt{n}}{8 k}+1.15 \cdot \frac{(n-k) \sqrt{n}}{k^{3 / 2}}+\frac{3 \sqrt{n}}{k}\right\} .
\end{aligned}
$$

We assumed that $n-\frac{0.4 \cdot n}{\log n+1.02} \leq k$ and $n \geq 5000$. By using the inequality $e^{-x}<\frac{1}{1+x}$ we obtain that $n e^{-\frac{0.2}{\frac{\log n}{2}+0.31}}<n \cdot \frac{1}{1+\frac{10.2}{\frac{\log n}{2}+0.31}}=n-\frac{0.4 \cdot n}{\log n+1.02} \leq k$. As $n \geq 5000$, we have that $\frac{k}{n}>0.958$. 
By easy calculation from these inequalities the following ones can be deduced:

$$
\begin{gathered}
\left(\frac{\log n}{2}+0.31\right)(\log n-\log k)<0.2 \\
\frac{n+2 \sqrt{n}}{8 k}<0.135 \\
1.15 \cdot \frac{(n-k) \sqrt{n}}{k^{3 / 2}}+\frac{3 \sqrt{n}}{k}<0.096 .
\end{gathered}
$$

Adding (24), 25) and (26) using (23) we arrive at:

$$
S \leq k(0.2+0.135+0.096)=0.431 \cdot k .
$$

Then from inequalities (12), 13) and (15) in case $k / n>0.958$ we get

$$
|\underline{k} * \underline{n}| \geq k+2 k-n-2 S \geq 2.138 \cdot k-n>n,
$$

thus we proved the statement in Case 3 as well.

We proved the statement for all pairs $n, k$ where $n \geq 5000$. Cases $k \leq n \leq 5000$ can be checked by brute force.

\section{References}

P. Dusart. The $k$ th prime is greater than $k(\ln k+\ln \ln k-1)$ for $k \geq 2$. Math. Comp., 68(225):411-415, 1999.

R. Eggetsberger. On constructing codes from planar nearrings. http://www algebra uni-linz . ac.at/Nearrings/nrcodes.html, 2011.

G. Pilz. Near-rings, volume 23 of North-Holland Mathematics Studies. North-Holland Publishing Co., Amsterdam, second edition, 1983. ISBN 0-7204-0566-1. The theory and its applications.

G. Pilz. On polynomial near-ring codes. In Contributions to general algebra, 8 (Linz, 1991), pages 233-238. Hölder-Pichler-Tempsky, Vienna, 1992.

G. Pilz. Near-rings: What they are and what they are good for. http://www. algebra . uni-linz . ac.at/Nearrings/what-are.html, 2011.

G. Robin. Estimation de la fonction de Tchebychef $\theta$ sur le $k$-ième nombre premier et grandes valeurs de la fonction $\omega(n)$ nombre de diviseurs premiers de $n$. Acta Arith., 42(4):367-389, 1983.

J. B. Rosser and L. Schoenfeld. Approximate formulas for some functions of prime numbers. Illinois J. Math., 6:64-94, 1962. 
Péter Pál Pach and Csaba Szabó 